\title{
GLOBAL PROPERTIES OF THE GAUSS IMAGE OF FLAT SURFACES IN $R^{4}$
}

\author{
By KaZUYUKI ENOMOTO
}

Let $M$ be a surface of zero Gaussian curvature in $R^{4}$ which has flat normal connection. Let $G_{2,4}$ denote the Grassmann manifold consisting of oriented 2-dimensional linear subspaces of $R^{4}$. The Gauss map $G: M \rightarrow G_{2,4}$ is defined by assigning each point of $M$ to the tangent plane of $M$ at the point. The image of $M$ by $G$ is called the Gauss image of $M$.

In [2] we studied local properties of the Gauss image of $M$; if we identify $G_{2,4}$ with $S^{2} \times S^{2}$, then the Gauss image of $M$ is locally the Riemannian product of two curves $\gamma_{1}$ and $\gamma_{2}$, where $\gamma_{1}$ lies in the first factor of $S^{2} \times S^{2}$ and $\gamma_{2}$ lies in the second factor.

In this paper we study some global properties of the Gauss image of $M$ when $M$ is compact. If $G$ is regular at every point of $M$, the Gauss image is a finite covering of $\gamma_{1} \times \gamma_{2}$, where $\gamma_{1}$ (resp. $\gamma_{2}$ ) is a closed curve in the first (resp. second) factor of $G_{2,4}$. Then we show that the total curvatures of $\gamma_{1}$ and $\gamma_{2}$ are both zero (Theorem 1). In particular, if $\gamma_{2}$ is simple, it divides the factor of $G_{2,4}\left(=S^{2}(1 / \sqrt{ } 2)\right)$ into two regions of the same area.

In $\S 3$, we give a method to construct a flat torus whose Gauss image is prescribed. In Theorem 2, we show that if $\gamma_{2}(i=1,2)$ is a closed curve in $S^{2}(1 / \sqrt{ } 2)$ whose total curvature is zero and if the total curvature of any subarc of $\gamma_{2}$ is less than $\pi / 2$, then there exists a flat torus whose Gauss image is a finite covering of $\gamma_{1} \times \gamma_{2}$.

The author would like to thank Joel Weiner for his helpful comments on this paper.

\section{$\S 1$. Local Properties.}

In this section, we recall some basic facts on the geometry of the Grassmann manifold $G_{2,4}$ and flat surfaces in $R^{4}$. See [2] for details. Let $G_{2,4}$ denote the Grassmann manifold of oriented 2-dimensional linear subspaces of $R^{4}$. If $P \in G_{2,4}$, then there exists a positively oriented orthonormal basis $\left\{e_{1}, e_{2}, e_{3}, e_{4}\right\}$ of $R^{4}$ such that $P=e_{1} \wedge e_{2}$. By differentiating $e_{1} \wedge e_{2}$, we see that the tangent space of $G_{2,4}$ at $P, T_{P} G_{2,4}$, is spanned by $e_{2} \wedge e_{\alpha}(i=1,2, \alpha=3,4)$. It is known that $G_{2,4}$ equipped with the standard invariant metric is isometric to $S^{2}(1 / \sqrt{ } 2) \times S^{2}(1 / \sqrt{ } 2)$

Received December 1, 1986. 
([3]). We identify $G_{2,4}$ with $S^{2} \times S^{2}$ and denote the first factor by $S_{1}$ and the second by $S_{2} . \quad\left\{e_{\imath} \wedge e_{\alpha} ; i=1,2, \alpha=3,4\right\}$ becomes an orthonormal basis of $T_{P} G_{2,4}$ with respect to this metric, and $T_{P} S_{1}$ is spanned by $\left\{e_{1} \wedge e_{3}-e_{2} \wedge e_{4}, e_{1} \wedge e_{4}+e_{2} \wedge e_{3}\right\}$ and $T_{P} S_{2}$ is spanned by $\left\{e_{1} \wedge e_{3}+e_{2} \wedge e_{4}, e_{1} \wedge e_{4}-e_{2} \wedge e_{3}\right\}$.

Let $M$ be an oriented surface in $R^{4}$. The Gauss map $G: M \rightarrow G_{2,4}$ is defined as $G(p)=T_{p} M$, where $T_{p} M$ is the tangent plane of $M$ at $p$. Let $\left\{e_{1}, e_{2}, e_{3}, e_{4}\right\}$ be a positively oriented orthonormal frame field of $R^{4}$ which is defined on an open set of $M$ and satisfies $T_{p} M=e_{1}(p) \wedge e_{2}(p)$. Let $\omega_{A B}(1 \leqq A, B \leqq 4)$ be the connection form of the standard covariant differentiation of $R^{4}$, i.e., $\omega_{A B}=$ $\left\langle d e_{A}, e_{B}\right\rangle$, where $\langle$,$\rangle is the standard inner product of R^{4}$. The differential of the Gauss map is given by

$$
d G(X)=-\omega_{13}(X) e_{2} \wedge e_{3}-\omega_{14}(X) e_{2} \wedge e_{4}+\omega_{23}(X) e_{1} \wedge e_{3}+\omega_{24}(X) e_{1} \wedge e_{4}
$$

for any $X \in T_{p} M$.

LEMMA 1.1. ([2]) If the Gaussian curvature of $M$ is identically zero and the normal connection of $M$ is flat, then there exists a local orthonormal frame fleld $\left\{e_{1}, e_{2}, e_{3}, e_{4}\right\}$ on $M$ such that $e_{1}(p) \wedge e_{2}(p)=T_{p} M$ for any $p \in M, \omega_{14}(X)=\omega_{23}(X)=0$ for any $X \in T_{p} M$ and $\omega_{13}\left(e_{2}\right)=\omega_{24}\left(e_{1}\right)=0$.

Let $\lambda=\omega_{13}\left(e_{1}\right)$ and $\mu=\omega_{24}\left(e_{2}\right)$. Then (1.1) is written as

$$
d G(X)=-\lambda\left\langle X, e_{1}\right\rangle e_{2} \wedge e_{3}+\mu\left\langle X, e_{2}\right\rangle e_{1} \wedge e_{4} .
$$

Hence $G$ is regular at $p M$ if and only if $\lambda \mu \neq 0$. Moreover, if $\lambda \mu \neq 0$ on $M$, then there is no umbilical point on $M$ and it follows from Reckziegel's theorem ([5]) that each $e_{A}(1 \leqq A \leqq 4)$ becomes a $C^{\infty}$ vector field, and $\lambda$ and $\mu$ become $C^{\infty}$ functions on $M$. Applying Codazzi equations, we obtain the following equalities :

$$
\begin{aligned}
& e_{1} \mu-\mu \omega_{21}\left(e_{2}\right)=0 \\
& e_{2} \lambda-\lambda \omega_{12}\left(e_{1}\right)=0 \\
& \lambda \omega_{21}\left(e_{2}\right)-\mu \omega_{34}\left(e_{1}\right)=0 \\
& \mu \omega_{12}\left(e_{1}\right)+\lambda \omega_{34}\left(e_{2}\right)=0 .
\end{aligned}
$$

In the following, we assume that $\lambda \mu \neq 0$ on $M$. Then there exists a local coordinate system $\left(\xi_{1}, \xi_{2}\right)$ on $M$ which satisfies

$$
\begin{aligned}
& \partial / \partial \xi_{1}=1 / \sqrt{ } 2\left(1 / \lambda e_{1}-1 / \mu e_{2}\right) \\
& \partial / \partial \xi_{2}=1 / \sqrt{ } 2\left(1 / \lambda e_{1}+1 / \mu e_{2}\right) .
\end{aligned}
$$

Let $\sigma_{1}\left(\right.$ resp. $\left.\sigma_{2}\right)$ be an integral curve of $1 / \sqrt{ } 2\left(1 / \lambda e_{1}-1 / \mu e_{2}\right)\left(\right.$ resp. $1 / \sqrt{ } 2\left(1 / \lambda e_{1}\right.$ $\left.\left.+1 / \mu e_{2}\right)\right)$. Let $\gamma_{\imath}=G\left(\sigma_{\imath}\right)(i=1,2)$. Then we have 
Proposition 1. ([2]) $\gamma_{\imath}$ lies in $S_{\imath}$ for each $i=1,2$. Locally, $G(M)$ is the Riemannian product of $\gamma_{1}$ and $\gamma_{2}$.

Let $\kappa_{\imath}$ denote the geodesic curvature of $\gamma_{\imath}(i=1,2)$ in $S_{\imath}=S^{2}(1 / \sqrt{ } 2)$. By Proposition 4.1 in [2], we have

$$
\begin{aligned}
& \kappa_{1}=\sqrt{ } 2\left(e_{1} \frac{1}{\mu}+e_{2} \frac{1}{\lambda}\right) \\
& \kappa_{2}=\sqrt{ } 2\left(e_{1} \frac{1}{\mu}-e_{2} \frac{1}{\lambda}\right)
\end{aligned}
$$

Examples. (i) Let $C_{1}$ and $C_{2}$ be plane curves. Then the Riemannian product $C_{1} \times C_{2}$ is a flat surface in $R^{4}$ with flat normal connection. For this surface we have $\kappa_{1} \equiv 0$ and $\kappa_{2} \equiv 0$. Thus the Gauss image is locally the product of two great circles. Conversely, if the Gauss image of a flat surface in $R^{4}$ is totally geodesic, then it is locally the Riemannian product of two plane curves. This follows from the Chen-Yamaguchi classification theorem for surfaces in $R^{4}$ with totally geodesic Gauss image ([1]).

(ii) Let $\left(z_{1}(t), z_{2}(t)\right)$ be a curve parameterized by arc-length in $C^{2}=R^{4}$. Suppose $\left|z_{1}\right|^{2}+\left|z_{2}\right|^{2} \equiv 1$ so that this curve is contained in $S^{3} \subset R^{4}$. In addition, $\left(z_{1}, z_{2}\right)$ is required to satisfy $z_{1}^{\prime} \overline{z_{1}}+z_{2}^{\prime} \overline{z_{2}} \equiv 0$. Let $M$ be the surface in $R^{4}$ defined by $(t, \phi) \rightarrow\left(e^{\imath \phi} z_{1}(t), e^{\imath \phi} z_{2}(t)\right)$. Then $M$ is a flat surface with flat normal connection. For this surface we have either $\kappa_{1} \equiv 0$ or $\kappa_{2} \equiv 0$. ( $M$ is called a Hopf torus. Some descriptions of the geometry of Hopf tori are given in [4].)

\section{§2. Global Properties.}

Let $M$ be a compact, oriented surface in $R^{4}$ which has zero Gaussian curvature and flat normal connection at every point. We assume that the Gauss map $G: M \rightarrow G_{2,4}=S_{1} \times S_{2}$ is regular at every point. Then it follows from Proposition 1 that there exist immersed closed curves $\gamma_{1} \subset S_{1}$ and $\gamma_{2} \subset S_{2}$ such that the Gauss image $G(M)$ is a finite covering of the Riemannian product of $\gamma_{1}$ and $\gamma_{2}$.

Combining (1.8) with (1.3), (1.4) and (1.7), we obtain

$$
\begin{aligned}
& \kappa_{1}(G(p))=-2 \omega_{12}\left(\partial / \partial \xi_{1}(p)\right) \\
& \kappa_{2}(G(p))=2 \omega_{12}\left(\partial / \partial \xi_{2}(p)\right),
\end{aligned}
$$

for any $p \in M$.

Since the Gaussian curvature is identically zero, the universal covering $\tilde{M}$ of $M$ is isometric to $R^{2}$ equipped with the standard flat metric. Let $\pi: \tilde{M} \rightarrow M$ be the projection. Since $M$ is compact, $M$ is isometric to $R^{2} / \Gamma$, where $\Gamma$ is a properly discontinuous subgroup of the isometry group of $R^{2}$. Let $\left(x_{1}, x_{2}\right)$ be a Cartesian coordinate system on $\tilde{M}$. Suppose that $\Gamma$ is generated by $\left(x_{1}, x_{2}\right) \rightarrow$ $\left(x_{1}+a, x_{2}\right)$ and $\left(x_{1}, x_{2}\right) \rightarrow\left(x_{1}+b, x_{2}+c\right)$ for some real numbers $a, b$ and $c$. Let 
$f_{\imath}=d \pi\left(\partial / \partial x_{\imath}\right)(i=1,2)$. Then $\left\{f_{1}, f_{2}\right\}$ is a globally defined $C^{\infty}$ parallel orthonormal frame field of $T M$.

There exists a global $C^{\infty}$ orthonormal frame field $\left\{\tilde{e}_{1}, \tilde{e}_{2}\right\}$ on $\tilde{M}$ such that $e_{1}=d \pi\left(\tilde{e}_{1}\right)$ and $e_{2}=d \pi\left(\tilde{e}_{2}\right)$ satisfy the conditions in Lemma 1.1. Let $\tilde{\lambda}=\lambda \circ \pi$ and $\tilde{\mu}=\mu \circ \pi$. Then $\tilde{\lambda}$ and $\tilde{\mu}$ are non-zero $C^{\infty}$ functions on $\tilde{M}$. Let $\tilde{X}_{1}=1 / \sqrt{ } 2\left(1 / \tilde{\lambda} \tilde{e}_{1}\right.$ $\left.-1 / \tilde{\mu} \tilde{e}_{2}\right)$ and $\tilde{X}_{2}=1 / \sqrt{ } 2\left(1 / \tilde{\lambda} \tilde{e}_{1}+1 / \tilde{\mu} \tilde{e}_{2}\right)$. Then we have $\left[\tilde{X}_{1}, \tilde{X}_{2}\right]=0$ everywhere on $M$. Hence there exists a global coordinate system $\left(\tilde{\xi}_{1}, \tilde{\xi}_{2}\right)$ on $\tilde{M}$ such that $\partial / \partial \tilde{\xi}_{1}=\tilde{X}_{1}$ and $\partial / \partial \tilde{\xi}_{2}=\tilde{X}_{2}$.

We define a $C^{\infty}$ function $\alpha$ on $\tilde{M}$ by

$$
\begin{aligned}
& \tilde{e}_{1}=\cos \alpha \partial / \partial x_{1}+\sin \alpha \partial / \partial x_{2} \\
& \tilde{e}_{2}=-\sin \alpha \partial / \partial x_{1}+\cos \alpha \partial / \partial x_{2} .
\end{aligned}
$$

Then

$$
\begin{aligned}
\pi^{*} \omega_{12} & =\left\langle d \tilde{e}_{1}, \tilde{e}_{2}\right\rangle \\
& =d \alpha .
\end{aligned}
$$

For a curve $\gamma$ in a two dimensional oriented Riemannian manifold the total integral of the signed geodesic curvature $\kappa$ along $\gamma, \int_{\gamma} \kappa$, is called the total curvature of $\gamma$. We denote it by $\tau(\gamma)$.

Let $\tilde{\sigma}_{1}=\left\{\left(\tilde{\xi}_{1}, \tilde{\xi}_{2}\right):-\infty<\tilde{\xi}_{1}<\infty, \tilde{\xi}_{2} \equiv 0\right\}$ and $\tilde{\sigma}_{2}=\left\{\left(\tilde{\xi}_{1}, \tilde{\xi}_{2}\right): \tilde{\xi}_{1} \equiv 0,-\infty<\tilde{\xi}_{2}<\infty\right\}$. $\tilde{\sigma}_{1}$ and $\tilde{\sigma}_{2}$ are curves on $\tilde{M}$ and $G \circ \pi$ maps $\tilde{\sigma}_{\imath}$ onto a closed curve $\gamma_{2}$ in $S_{\imath}$ for $i=1,2$. Since $G$ is regular everywhere on $M$ and $M$ is compact, $G(M)$ is a finite covering of $\gamma_{1} \times \gamma_{2}$. Let $k$ be the degree of $G$ as a map from $M$ onto $\gamma_{1} \times \gamma_{2}$. There exists a closed curve $\sigma_{\imath}$ on $M$ such that $\tilde{\sigma}_{\imath}$ is mapped onto $\sigma_{\imath}$ by $\pi$ and $G\left(\sigma_{\imath}\right)$ is a $k$-fold covering of $\gamma_{\imath}$.

LEMMA 2.1. For $i=1,2$, we have

$$
\tau\left(\sigma_{\imath}\right)=\frac{(-1)^{\imath} k}{2} \tau\left(\gamma_{\imath}\right)
$$

Proof. Let $\tilde{\sigma}_{\imath}^{\prime}$ be a subarc of $\tilde{\sigma}_{\imath}$ which is mapped bijectively onto $\sigma_{\imath}$ by $\pi$. Then we have $\tau\left(\tilde{\sigma}_{\imath}^{\prime}\right)=\tau\left(\sigma_{\imath}\right)$.

On the other hand, by (2.1) and (2.3), we have

$$
\tau\left(\gamma_{\imath}\right)=\int_{\gamma_{i}} \kappa_{\imath}=\frac{(-1)^{i} 2}{k} \int_{\tilde{\sigma}_{i}^{\prime}} d \alpha=\frac{(-1)^{i} 2}{k} \tau\left(\tilde{\sigma}_{\imath}^{\prime}\right) . \quad \text { Q.E.D. }
$$

LEMMA 2.2. Let $\sigma$ be a smooth closed curve in a flat torus $M=R^{2} / \Gamma$. Let $\tilde{\sigma}$ be a complete curve in the universal covering $\tilde{M}=R^{2}$ of $M$ whose image by the projection $\pi: \tilde{M} \rightarrow M$ is $\sigma$.

If the total curvature of $\sigma$ is not zero, then $\tilde{\sigma}$ has a self-intersection. 
Proof. Let $s$ be an arc-length parameter of $\sigma$ and let $L$ be the total length of $\sigma$. Let $s$ also denote an arc-length parameter of $\tilde{\sigma}$. For any $s$ and any integer $m$ we have $\pi \circ \tilde{\sigma}(s+m L)=\pi \circ \tilde{\sigma}(s)$. Suppose that $\Gamma$ is generated by $\left(x_{1}, x_{2}\right) \rightarrow\left(x_{1}+a, x_{2}\right)$ and $\left(x_{1}, x_{2}\right) \rightarrow\left(x_{1}+b, x_{2}+c\right)$. Since $\pi \circ \tilde{\sigma}(s)=\pi \circ \tilde{\sigma}(s+L)$, if $\tilde{\sigma}(s)=\left(x_{1}, x_{2}\right)$, there exist integers $p$ and $q$ such that $\tilde{\sigma}(s+L)=\left(x_{1}+p a+q b\right.$, $\left.x_{2}+q c\right)$.

Let $\kappa(s)(0 \leqq s<L)$ be the signed geodesic curvature of $\sigma$. We extend $\kappa$ to a function defined on $(-\infty, \infty)$ by setting $\kappa(s)=\kappa\left(s^{\prime}\right)$ if $s \equiv s^{\prime}(\bmod L)$ and $s^{\prime} \in[0, L)$. Then $\kappa(s)$ gives the signed geodesic curvature of $\tilde{\sigma}$. We define a function $F(s)$ for $s \in(-\infty, \infty)$ by

$$
F(s)=\int_{0}^{s} \kappa(t) d t
$$

so that the total curvature of $\left\{\tilde{\sigma}(s): s_{1} \leqq s \leqq s_{2}\right\}$ is given by $F\left(s_{1}\right)-F\left(s_{2}\right)$. In particular, for any $s$, we have $F(s+L)-F(s)=\tau(\sigma)$.

Let $\Lambda_{1}=\min \{F(s): 0 \leqq s \leqq L\}$ and $\Lambda_{2}=\max \{F(s): 0 \leqq s \leqq L\}$. Suppose $\tau(\sigma)=$ $A \neq 0$. Then for any $s_{1}$ and $s_{2}\left(s_{1}<s_{2}\right)$ we have

$$
m A+\Lambda_{1} \leqq F\left(s_{2}\right)-F\left(s_{1}\right) \leqq m A+\Lambda_{2},
$$

where $m$ is the integer such that $m \leqq s_{2}-s_{1}<m+1$. Therefore, there exist numbers $S_{1}$ and $S_{2}$ such that

$$
\left|F\left(s_{2}\right)-F\left(s_{1}\right)\right|>2 \pi
$$

for any $s_{1}<S_{1}$ and $s_{2}>S_{2}$.

Since $\left\{\tilde{\sigma}(s): S_{1}<s<S_{2}\right\}$ is bounded, there exists a circle $C$ in $\tilde{M}$ which contains $\left\{\tilde{\sigma}(s): S_{1}<s<S_{2}\right\}$ inside. If $\tilde{\sigma}(s)=\left(x_{1}, x_{2}\right)$, we have $\tilde{\sigma}(s+n L)=$ $\left(x_{1}+n p a+n q b, x_{2}+n q c\right)$. We see from this that the whole $\tilde{\sigma}$ is not bounded and there exist numbers $S_{3}<S_{1}$ and $S_{4}>S_{2}$ such that $\tilde{\sigma}\left(S_{3}\right)$ and $\tilde{\sigma}\left(S_{4}\right)$ lie outside $C$. Then $\sigma$ must intersect $C$ at some $\tilde{\sigma}\left(T_{1}\right)$ and $\tilde{\sigma}\left(T_{2}\right)$, where $S_{3}<T_{1}<S_{1}$ and $S_{2}<T_{2}<S_{4}$.

If $\tilde{\sigma}$ does not have a self-intersection, then $\left\{\tilde{\sigma}(s): T_{1} \leqq s \leqq T_{2}\right\}$ divides the domain bounded by $C$ into two simply connected subdomains. By the GaussBonnet theorem, the total curvature of $\left\{\sigma(s): T_{1} \leqq s \leqq T_{2}\right\}$ is smaller than $2 \pi$. Thus we obtain $\left|F\left(T_{2}\right)-F\left(T_{1}\right)\right|<2 \pi$, which contradicts (2.4). Hence $\tilde{\sigma}$ must have a self-intersection. Q.E.D.

THEOREM 1. Let $M$ be a compact, oriented surface in $R^{4}$ which has zero Gaussian curvature and flat normal connection. Suppose that the Gauss map $G$ is regular at every point of $M$. Then there exist closed curves $\gamma_{1} \subset S_{1}$ and $\gamma_{2} \subset S_{2}$ such that the Gauss image $G(M)$ is a finite covering of the Riemannian product of $\gamma_{1}$ and $\gamma_{2}$ and the total curvature of each $\gamma_{2}$ is zero for $i=1,2$.

Proof. Since $\left(\tilde{\xi}_{1}, \tilde{\xi}_{2}\right)$ defines a global coordinate system on $\tilde{M}, \tilde{\sigma}_{\imath}=$ $\left\{\left(\tilde{\xi}_{1}, \tilde{\xi}_{2}\right): \tilde{\xi}_{i} \equiv 0\right\}$ does not have a self-intersection. Since $\sigma_{\imath}=\pi \circ \tilde{\sigma}_{\imath}$ is closed, we 
use Lemma 2.2 to see that $\tau\left(\sigma_{\imath}\right)=0$. Now we have $\tau\left(\gamma_{\imath}\right)=0$ by Lemma 2.1 . Q.E.D.

COROLLARY. If $\gamma_{\imath}$ is a simple closed curve in $S_{\imath}, \gamma_{\imath}$ divides $S_{\imath}$ into two domains of the same area.

Proof. Since the total curvature of $\gamma_{2}$ is zero, the Gauss-Bonnet theorem implies the corollary.

Remark. If $M$ is the product of two plane curves $C_{1} \times C_{2}$, then $G(M)$ is a double covering of the product of two great circles. If $M$ is a Hopf torus, then $G(M)$ is a double covering of the product of a great circle in one of $S_{1}$ and $S_{2}$ and a closed curve in the other.

\section{§ 3. Flat Tori with Prescribed Gauss Images.}

In this section, we prove the following theorem. Again, we identify $G_{2,4}$ with $S_{1} \times S_{2}$, where $S_{1}$ and $S_{2}$ are isometric to a round 2 -sphere of radius $1 / \sqrt{ } 2$.

TheOREM 2. Let $\gamma_{\imath}$ be a regular closed curve in $S_{\imath}(i=1,2)$. Suppose that the total curvature of $\gamma_{2}$ is zero and the total curvature of any subarc of $\gamma_{2}$ is less than $\pi / 2$. Then there exists a compact immersed surface in $R^{4}$ whose Gauss image is a finite covering of the Riemannian product of $\gamma_{1}$ and $\gamma_{2}$.

Remark. Local existence of a surface in $R^{4}$ with prescribed Gauss image is studied by $\mathrm{J}$. Weiner ([6]).

Let $\gamma_{i}\left(\xi_{i}\right)$ be a closed curve on $S_{\imath}$ which is parameterized by arc-length. Let $l_{\imath}$ be the total length of $\gamma_{i}$. We assume that $\gamma_{i}\left(\xi_{i}\right)$ is defined for all $\xi_{i}$ so that $\gamma_{i}\left(\xi_{i}+l_{2}\right)=\gamma_{i}\left(\xi_{i}\right)$ for any $\xi_{i}$. We denote the signed geodesic curvature of $\gamma_{\imath}$ by $\kappa_{\imath}$. Then our conditions for $\gamma_{\imath}$ are written as

$$
\begin{aligned}
& \int_{\gamma_{i}} \kappa_{i}\left(\xi_{i}\right) d \xi_{i}=0 \\
& \left|\int_{\gamma_{i}^{\prime}} \kappa_{i}\left(\xi_{i}\right) d \xi_{i}\right|<\frac{\pi}{2},
\end{aligned}
$$

where $\gamma_{i}^{\prime}$ is any subarc of $\gamma_{2}$.

We define a map $P: R^{2} \rightarrow G_{2,4}$ by $P\left(\xi_{1}, \xi_{2}\right)=\left(\gamma_{1}\left(\xi_{1}\right), \gamma_{2}\left(\xi_{2}\right)\right) \in S_{1} \times S_{2}=G_{2,4}$. Since $\gamma_{i}\left(\xi_{i}+m l_{i}\right)=\gamma_{i}\left(\xi_{i}\right)$ for any integer $m$, we have

$$
P\left(\xi_{1}+m_{1} l_{1}, \xi_{2}+m_{2} l_{2}\right)=P\left(\xi_{1}, \xi_{2}\right)
$$

for any integers $m_{1}, m_{2}$ and any $\xi_{1}, \xi_{2}$.

Let $\left\{\vec{e}_{A}\left(\xi_{1}, \xi_{2}\right): A=1,2,3,4\right\}$ be a set of $R^{4}$-valued functions which are defined on $R^{2}$ and satisfy the following conditions; 


$$
\left\langle\bar{e}_{A}\left(\xi_{1}, \xi_{2}\right), \bar{e}_{B}\left(\xi_{1}, \xi_{2}\right)\right\rangle=\delta_{A B} \text { for all } A, B=1, \cdots, 4
$$

$$
P\left(\xi_{1}, \xi_{2}\right)=\bar{e}_{1}\left(\xi_{1}, \xi_{2}\right) \wedge \bar{e}_{2}\left(\xi_{1}, \xi_{2}\right)
$$

$$
\left\{\bar{e}_{1}, \bar{e}_{2}, \bar{e}_{3}, \bar{e}_{4}\right\} \text { is positively oriented in } R^{4}
$$

for all $\left(\xi_{1}, \xi_{2}\right)$ in $R^{2}$.

Let $\bar{\omega}_{A B}$ be a 1 -form on $R^{2}$ which is defined by $\bar{\omega}_{A B}=\left\langle d \bar{e}_{A}, \bar{e}_{B}\right\rangle$

LEMMA 3.1.

$$
\begin{array}{ll}
\bar{\omega}_{23}\left(\partial / \partial \xi_{1}\right)=\bar{\omega}_{14}\left(\partial / \partial \xi_{1}\right), & \bar{\omega}_{24}\left(\partial / \partial \xi_{1}\right)=-\bar{\omega}_{13}\left(\partial / \partial \xi_{1}\right) \\
\bar{\omega}_{23}\left(\partial / \partial \xi_{2}\right)=-\bar{\omega}_{14}\left(\partial / \partial \xi_{2}\right), & \bar{\omega}_{24}\left(\partial / \partial \xi_{2}\right)=\bar{\omega}_{13}\left(\partial / \partial \xi_{2}\right)
\end{array}
$$

Proof. Differentiating $\bar{e}_{1} \wedge \bar{e}_{2}$, we have

$$
d\left(\bar{e}_{1} \wedge \bar{e}_{2}\right)=\bar{\omega}_{23} \bar{e}_{1} \wedge \bar{e}_{3}+\bar{\omega}_{24} \bar{e}_{1} \wedge \bar{e}_{4}-\bar{\omega}_{13} \bar{e}_{2} \wedge \bar{e}_{3}-\bar{\omega}_{14} \bar{e}_{2} \wedge \bar{e}_{4} .
$$

Since $d\left(\bar{e}_{1} \wedge \bar{e}_{2}\right)\left(\partial / \partial \xi_{1}\right)$ is tangent to $\gamma_{1}$ in $S_{1}, d\left(\bar{e}_{1} \wedge \bar{e}_{2}\right)\left(\partial / \partial \xi_{1}\right)$ is a linear combination of $\bar{e}_{1} \wedge \bar{e}_{3}-\bar{e}_{2} \wedge \bar{e}_{4}$ and $\bar{e}_{1} \wedge \bar{e}_{4}+\bar{e}_{2} \wedge \bar{e}_{3}$. This yields the first two equations. Similarly, the last two equations follow from the fact that $d\left(\bar{e}_{1} \wedge \bar{e}_{2}\right)\left(\partial / \partial \xi_{2}\right)$ is tangent to $S_{2}$. Q.E. D.

Set

$$
\begin{array}{ll}
e_{1}=\cos \theta \bar{e}_{1}+\sin \theta \bar{e}_{2}, & e_{2}=-\sin \theta \bar{e}_{1}+\cos \theta \bar{e}_{2} \\
e_{3}=\cos \phi \bar{e}_{3}+\sin \phi \bar{e}_{4}, & e_{4}=-\sin \phi \bar{e}_{3}+\cos \phi \bar{e}_{4} .
\end{array}
$$

Let $\omega_{A B}=\left\langle d e_{A}, e_{B}\right\rangle$. Then we have the following lemma by an easy computation.

LEMMA 3.2.

$$
\begin{aligned}
& \omega_{14}\left(\partial / \partial \xi_{1}\right)=-\bar{\omega}_{13}\left(\partial / \partial \xi_{1}\right) \sin (\theta+\phi)+\bar{\omega}_{14}\left(\partial / \partial \xi_{1}\right) \cos (\theta+\phi) \\
& \omega_{14}\left(\partial / \partial \xi_{2}\right)=\bar{\omega}_{13}\left(\partial / \partial \xi_{2}\right) \sin (\theta-\phi)+\bar{\omega}_{14}\left(\partial / \partial \xi_{2}\right) \cos (\theta-\phi)
\end{aligned}
$$

We need the following lemma to prove Lemma 3.4. The proof is also easy.

LEMMA 3.3. Let $f\left(\xi_{1}, \xi_{2}\right)$ and $g\left(\xi_{1}, \xi_{2}\right)$ be $C^{\infty}$ functions on $R^{2}$. If $f^{2}+g^{2}$ never vanishes on $R^{2}$, then there exists a $C^{\infty}$ function $\alpha\left(\xi_{1}, \xi_{2}\right)$ defined on $R^{2}$ such that

$$
f\left(\xi_{1}, \xi_{2}\right) \cos \alpha\left(\xi_{1}, \xi_{2}\right)+g\left(\xi_{1}, \xi_{2}\right) \sin \alpha\left(\xi_{1}, \xi_{2}\right)=0
$$

for all $\left(\xi_{1}, \xi_{2}\right)$ in $R^{2}$.

LEMMA 3.4. There exist $C^{\infty}$ maps $e_{A}: R^{2} \rightarrow R^{4}(A=1, \cdots, 4)$ which satisfy the following conditions: 


$$
\begin{aligned}
& \left\langle e_{A}\left(\xi_{1}, \xi_{2}\right), e_{B}\left(\xi_{1}, \xi_{2}\right)\right\rangle=\delta_{A B} \text { for all } A, B=1, \cdots, 4 \\
& P\left(\xi_{1}, \xi_{2}\right)=e_{1}\left(\xi_{1}, \xi_{2}\right) \wedge e_{2}\left(\xi_{1}, \xi_{2}\right) \\
& \left\{e_{1}, e_{2}, e_{3}, e_{4}\right\} \text { is positively oriented in } R^{4} \\
& \omega_{14} \equiv 0 .
\end{aligned}
$$

Proof. Since $\left.d\left(e_{1} \wedge e_{2}\right)\left(\partial / \partial \xi_{i}\right) \neq 0, \quad\left(\bar{\omega}_{13}\left(\partial / \partial \xi_{2}\right)\right)^{2}+\bar{\omega}_{14}\left(\partial / \partial \xi_{i}\right)\right)^{2} \neq 0$ by Lemma 3.1. Hence, by Lemma 3.3, there exist $C^{\infty}$ functions $\alpha_{i}\left(\xi_{1}, \xi_{2}\right)$ defined on $R^{2}$ such that

$$
\begin{array}{r}
-\bar{\omega}_{13}\left(\partial / \partial \xi_{1}\right) \sin \alpha_{1}+\bar{\omega}_{14}\left(\partial / \partial \xi_{1}\right) \cos \alpha_{1} \equiv 0 \\
\bar{\omega}_{13}\left(\partial / \partial \xi_{2}\right) \sin \alpha_{2}+\bar{\omega}_{14}\left(\partial / \partial \xi_{2}\right) \cos \alpha_{2} \equiv 0 .
\end{array}
$$

Set $\theta=\left(\alpha_{1}+\alpha_{2}\right) / 2$ and $\phi=\left(\alpha_{1}-\alpha_{2}\right) / 2$. Then $\left\{e_{A}\left(\xi_{1}, \xi_{2}\right): A=1, \cdots, 4\right\}$ defined by (3.7) is a set of $C^{\infty}$ maps from $R^{2}$ to $R^{4}$ which satisfies the conditions (3.8)(3.11). Q.E.D.

Such a special set of vectors as $\left\{e_{A}: A=1, \cdots, 4\right\}$ in Lemma 3.4 is not unique but very limited as we see in the following lemma;

LEMMA 3.5. Let $\left\{e_{A}: A=1, \cdots, 4\right\}$ be a set of $R^{4}$-valued functions defined on $R^{2}$ which satisfies the conditions in Lemma 3.4. Let $\left\{e_{A}^{\prime}: A=1, \cdots, 4\right\}$ be any other set of $R^{4}$-valued functions satisfying (3.8)-(3.11). Then $\left\{e_{1}^{\prime}, e_{2}^{\prime}, e_{3}^{\prime}, e_{4}^{\prime}\right\}$ must be one of the followings:

$$
\begin{array}{ll}
\text { (i ) }\left\{e_{1}, e_{2}, e_{3}, e_{4}\right\} & \text { (ii) }\left\{e_{1}, e_{2},-e_{3},-e_{4}\right\} \\
\text { (iii) }\left\{e_{2},-e_{1}, e_{4},-e_{3}\right\} & \text { (iv) }\left\{e_{2},-e_{1},-e_{4}, e_{3}\right\} \\
\text { (v) }\left\{-e_{1},-e_{2}, e_{3}, e_{4}\right\} & \text { (vi) }\left\{-e_{1},-e_{2},-e_{3},-e_{4}\right\} \\
\text { (vii) }\left\{-e_{2}, e_{1}, e_{4},-e_{3}\right\} & \text { (viii) }\left\{-e_{2}, e_{1},-e_{4}, e_{3}\right\}
\end{array}
$$

Proof. We may write

$$
\begin{array}{ll}
e_{1}^{\prime}=\cos \theta e_{1}+\sin \theta e_{2}, & e_{2}^{\prime}=-\sin \theta e_{1}+\cos \theta e_{2} \\
e_{3}^{\prime}=\cos \phi e_{3}+\sin \phi e_{4}, & e_{4}^{\prime}=-\sin \theta e_{3}+\cos \theta e_{4} .
\end{array}
$$

Then, by Lemma 3.2, we see that $\sin (\theta+\phi)=0$ and $\sin (\theta-\phi)=0$. Hence $\theta-\phi$ $=m \pi, \theta+\phi=n \pi$ for some integers $m, n$. Q. E. D.

LEMMA 3.6. There exists a $C^{\infty}$ function $\beta\left(\xi_{1}, \xi_{2}\right)$ defined on $R^{2}$ which satisfies $d \beta=\omega_{34}$.

Proof. Let $\left\{e_{A}: A=1, \cdots, 4\right\}$ be a set of $R^{4}$-valued functions given in Lemma 3.4. By Lemma 3.1, the condition $\omega_{14} \equiv 0$ implies $\omega_{23} \equiv 0$. Using the structure equation, we obtain 


$$
d \omega_{34}=\omega_{31} \wedge \omega_{14}+\omega_{32} \wedge \omega_{24} \equiv 0 .
$$

Since $\omega_{34}$ is a globally defined 1 -form on $R^{2}$, there exists a function $\beta$ on $R^{2}$ such that $d \beta=\omega_{34}$. Q.E. D.

LEMma 3.7. Let $\left\{e_{A}\left(\xi_{1}, \xi_{2}\right): A=1, \cdots, 4\right\}$ be a set of $R^{4}$-valued functions satisfying the conditions (3.8)-(3.11) in Lemma 3.4. Let $\kappa_{i}\left(\xi_{i}\right)$ be the signed geodesic curvature of $\gamma_{i}$. Then we have

$$
\kappa_{\imath}=-2(-1)^{i} \omega_{34}\left(\partial / \partial \xi_{i}\right)=-2(-1)^{i} \partial \beta / \partial \xi_{i} .
$$

Proof. By Lemma 3.1, we have $d\left(e_{1} \wedge e_{2}\right)\left(\partial / \partial \xi_{1}\right)=\omega_{24}\left(\partial / \partial \xi_{1}\right)\left(e_{1} \wedge e_{4}+e_{2} \wedge e_{3}\right)$. Since $\xi_{1}$ is an arc-length parameter of $\gamma_{1},\left\|d\left(e_{1} \wedge e_{2}\right)\left(\partial / \partial \xi_{1}\right)\right\|=1$. This implies $\omega_{24}\left(\partial / \partial \xi_{1}\right)= \pm 1 / \sqrt{ } 2$. Changing parameter from $\xi_{1}$ to $-\xi_{1}$ if necessary, we may assume that

$$
\omega_{24}\left(\partial / \partial \xi_{1}\right)=-1 / \sqrt{ } 2
$$

Using Lemma 3.1 again, we have

$$
\omega_{13}\left(\partial / \partial \xi_{1}\right)=1 / \sqrt{ } 2 .
$$

A similar argument shows that

$$
\omega_{24}\left(\partial / \partial \xi_{2}\right)=\omega_{13}\left(\partial / \partial \xi_{2}\right)=1 / \sqrt{ } 2 .
$$

Thus if we set $v_{i}=d\left(e_{1} \wedge e_{2}\right)\left(\partial / \partial \xi_{i}\right)$, then $v_{1}=-1 / \sqrt{ } 2\left(e_{1} \wedge e_{4}+e_{2} \wedge e_{3}\right), \quad v_{2}=$ $1 / \sqrt{ } 2\left(e_{1} \wedge e_{4}-e_{2} \wedge e_{3}\right)$. Note that $v_{i}$ is a unit tangent vector of $\gamma_{2}$. Let $\tilde{D}$ be the Riemannian connection on $G_{2,4}$ associated with the standard invariant metric. Using Lemma 3.1 in [2], we obtain $\widetilde{D} v_{1}=1 / \sqrt{ } 2\left(\omega_{12}+\omega_{34}\right)\left(e_{1} \wedge e_{3}-e_{2} \wedge e_{4}\right)$ and $\widetilde{D} v_{2}=1 / \sqrt{ } 2\left(\omega_{12}-\omega_{34}\right)\left(e_{1} \wedge e_{3}+e_{2} \wedge e_{4}\right)$. This gives

$$
\begin{aligned}
\kappa_{1} & =\left\langle\widetilde{D}_{\partial / \partial \xi_{1}} v_{1}, 1 / \sqrt{ } 2\left(e_{1} \wedge e_{3}-e_{2} \wedge e_{4}\right)\right\rangle \\
& =\omega_{12}\left(\partial / \partial \xi_{1}\right)+\omega_{34}\left(\partial / \partial \xi_{1}\right) \\
\kappa_{2} & =\left\langle\widetilde{D}_{\partial / \partial \xi_{2}} v_{2}, 1 / \sqrt{ } 2\left(e_{1} \wedge e_{3}+e_{2} \wedge e_{4}\right)\right\rangle \\
& =\omega_{12}\left(\partial / \partial \xi_{2}\right)-\omega_{34}\left(\partial / \partial \xi_{2}\right) .
\end{aligned}
$$

On the other hand, since $v_{i}$ is tangent to $S_{\imath}$, we have $\tilde{D}_{\partial / \partial \xi_{2}} v_{1}=0$ and $\tilde{D}_{\partial / \partial \xi_{1}} v_{2}=0$. This gives

$$
\begin{aligned}
& \omega_{12}\left(\partial / \partial \xi_{2}\right)+\omega_{34}\left(\partial / \partial \xi_{2}\right)=0 \\
& \omega_{12}\left(\partial / \partial \xi_{1}\right)-\omega_{34}\left(\partial / \partial \xi_{1}\right)=0 .
\end{aligned}
$$

Combining (3.16) and (3.17), we obtain 


$$
\begin{aligned}
& \kappa_{1}=2 \omega_{34}\left(\partial / \partial \xi_{1}\right)=2 \partial \beta / \partial \xi_{1} \\
& \kappa_{2}=-2 \omega_{34}\left(\partial / \partial \xi_{2}\right)=-2 \partial \beta / \partial \xi_{2} . \quad \text { Q. E. D. }
\end{aligned}
$$

By Lemma 3.7, the total curvature $\tau\left(\gamma_{1}\right)$ of $\gamma_{1}$ is given by

$$
\tau\left(\gamma_{1}\right)=\int_{\xi_{1}}^{\xi_{1}+l_{1}} 2 \partial \beta / \partial \xi_{1} d \xi_{1}=2\left(\beta\left(\xi_{1}+l_{1}, \xi_{2}\right)-\beta\left(\xi_{1}, \xi_{2}\right)\right),
$$

where $\left(\xi_{1}, \xi_{2}\right)$ is any point in $R^{2}$. Similarly, $\tau\left(\gamma_{2}\right)=-2\left(\beta\left(\xi_{1}, \xi_{2}+l_{2}\right)-\beta\left(\xi_{1}, \xi_{2}\right)\right)$. Thus we have the following lemma.

LEMMA 3.8. $\tau\left(\gamma_{1}\right)=0$ if and only if $\beta\left(\xi_{1}+l_{1}, \xi_{2}\right)=\beta\left(\xi_{1}, \xi_{2}\right) . \quad \tau\left(\gamma_{2}\right)=0$ if and only if $\beta\left(\xi_{1}, \xi_{2}+l_{2}\right)=\beta\left(\xi_{1}, \xi_{2}\right)$.

We define a $C^{\infty}$ map $x$ of $R^{2}$ into $S^{3}$ by $x\left(\xi_{1}, \xi_{2}\right)=-\cos \left(\beta\left(\xi_{1}, \xi_{2}\right)\right) e_{3}\left(\xi_{1}, \xi_{2}\right)$ $+\sin \left(\beta\left(\xi_{1}, \xi_{2}\right)\right) e_{4}\left(\xi_{1}, \xi_{2}\right)$, where $\beta\left(\xi_{1}, \xi_{2}\right)$ is the function on $R^{2}$ in Lemma 3.6 and $\left\{e_{3}, e_{4}\right\}$ is a set of $R^{4}$-valued functions defined on $R^{2}$ which is given in Lemma 3.4. Since $\gamma_{1}\left(\xi_{1}+l_{1}\right)=\gamma_{1}\left(\xi_{1}\right), P\left(\xi_{1}+l_{1}, \xi_{2}\right)=P\left(\xi_{1}, \xi_{2}\right)$. Hence, by Lemma 3.5 , we have only four possibilities for $\left\{e_{3}\left(\xi_{1}+l_{1}, \xi_{2}\right), e_{4}\left(\xi_{1}+l_{1}, \xi_{2}\right)\right\}$ as follows:

$$
\begin{aligned}
\left\{e_{3}\left(\xi_{1}+l_{1}, \xi_{2}\right), e_{4}\left(\xi_{1}+l_{1}, \xi_{2}\right)\right\} & =\left\{e_{3}\left(\xi_{1}, \xi_{2}\right), e_{4}\left(\xi_{1}, \xi_{2}\right)\right\} \\
\text { or } & =\left\{-e_{3}\left(\xi_{1}, \xi_{2}\right),-e_{4}\left(\xi_{1}, \xi_{2}\right)\right\} \\
\text { or } & =\left\{e_{4}\left(\xi_{1}, \xi_{2}\right),-e_{3}\left(\xi_{1}, \xi_{2}\right)\right\} \\
\text { or } & =\left\{-e_{4}\left(\xi_{1}, \xi_{2}\right), e_{3}\left(\xi_{1}, \xi_{2}\right)\right\}
\end{aligned}
$$

Note that, by continuity, if any of (a)-(d) holds for some $\left(\xi_{1}, \xi_{2}\right)$, it must hold for all $\left(\xi_{1}, \xi_{2}\right)$.

If we have (b), then $\left\{e_{3}\left(\xi_{1}+2 l_{1}, \xi_{2}\right), e_{4}\left(\xi_{1}+2 l_{1}, \xi_{2}\right)\right\}=\left\{e_{3}\left(\xi_{1}, \xi_{2}\right), e_{4}\left(\xi_{1}, \xi_{2}\right)\right\}$.

If we have (c) or (d), then $\left\{e_{3}\left(\xi_{1}+4 l_{1}, \xi_{2}\right), e_{4}\left(\xi_{1}+4 l_{1}, \xi_{2}\right)\right\}=\left\{e_{3}\left(\xi_{1}, \xi_{2}\right), e_{4}\left(\xi_{1}, \xi_{2}\right)\right\}$.

Summarizing these, we see that

$$
\left\{e_{3}\left(\xi_{1}+m_{1} l_{1}, \xi_{2}\right), e_{4}\left(\xi_{1}+m_{1} l_{1}, \xi_{2}\right)\right\}=\left\{e_{3}\left(\xi_{1}, \xi_{2}\right), e_{4}\left(\xi_{1}, \xi_{2}\right)\right\}
$$

holds for any $\left(\xi_{1}, \xi_{2}\right)$, where $m_{1}$ is 1,2 or 4 and constant for all $\left(\xi_{1}, \xi_{2}\right)$.

Similarly, it can be shown that

$$
\left\{e_{3}\left(\xi_{1}, \xi_{2}+m_{2} l_{2}\right), e_{4}\left(\xi_{1}, \xi_{2}+m_{2} l_{2}\right)\right\}=\left\{e_{3}\left(\xi_{1}, \xi_{2}\right), e_{4}\left(\xi_{1}, \xi_{2}\right)\right\}
$$

holds for any $\left(\xi_{1}, \xi_{2}\right)$, where $m_{2}$ is 1,2 or 4 and constant for all $\left(\xi_{1}, \xi_{2}\right)$.

If the total curvature of $\gamma_{i}$ is zero for $i=1,2$, we have

$$
\begin{aligned}
& \beta\left(\xi_{1}+l_{1}, \xi_{2}\right)=\beta\left(\xi_{1}, \xi_{2}\right) \\
& \beta\left(\xi_{1}, \xi_{2}+l_{2}\right)=\beta\left(\xi_{1}, \xi_{2}\right),
\end{aligned}
$$

by Lemma 3.8. 
Using (3.18), (3.19) and (3.20), we see that

$$
x\left(\xi_{1}+m_{1} l_{1}, \xi_{2}\right)=x\left(\xi_{1}, \xi_{2}\right)
$$

and

$$
x\left(\xi_{1}, \xi_{2}+m_{2} l_{2}\right)=x\left(\xi_{1}, \xi_{2}\right)
$$

(3.21) and (3.22) show that $x$ defines a $C^{\infty}$ map from a torus $R^{2} / \Gamma$ into $S^{3}$, where $\Gamma$ is a subgroup of $\operatorname{Isom}\left(R^{2}\right)$ which is generated by $\left(\xi_{1}, \xi_{2}\right) \rightarrow\left(\xi_{1}+m_{1} l_{1}, \xi_{2}\right)$ and $\left(\xi_{1}, \xi_{2}\right) \rightarrow\left(\xi_{1}, \xi_{2}+m_{2} l_{2}\right)$.

LEMMA 3.9. Let $x$ be the $C^{\infty}$ map from a torus into $S^{3}$ which is constructed above. Then $x$ is regular at $\left(\xi_{1}, \xi_{2}\right)$ if and only if $\beta\left(\xi_{1}, \xi_{2}\right) \neq k \pi / 2$ for any integer $k$.

Proof. The differential of $x$ is given by $d x=d\left(-\cos \beta e_{3}+\sin \beta e_{4}\right)=$ $\sin \beta d \beta e_{3}-\cos \beta\left(\omega_{31} e_{1}+\omega_{32} e_{2}+\omega_{34} e_{4}\right)+\cos \beta d \beta e_{4}+\sin \beta\left(\omega_{41} e_{1}+\omega_{42} e_{2}+\omega_{43} e_{3}\right)$.

Since $\omega_{23}\left(\partial / \partial \xi_{i}\right)=\omega_{14}\left(\partial / \partial \xi_{i}\right)=0$ by Lemma 3.4 and Lemma $3.1,-\omega_{24}\left(\partial / \partial \xi_{1}\right)=$ $\omega_{13}\left(\partial / \partial \xi_{1}\right)=1 / \sqrt{ } 2$ and $\omega_{24}\left(\partial / \partial \xi_{2}\right)=\omega_{13}\left(\partial / \partial \xi_{2}\right)=1 / \sqrt{ } 2$ by (3.13), (3.14) and (3.15), and $d \beta=\omega_{34}$ by Lemma 3.6 , we have

$$
d x\left(\partial / \partial \xi_{1}\right)=1 / \sqrt{ } 2\left(\cos \beta e_{1}+\sin \beta e_{2}\right)
$$

and

$$
d x\left(\partial / \partial \xi_{2}\right)=1 / \sqrt{ } 2\left(\cos \beta e_{1}-\sin \beta e_{2}\right) .
$$

From this, we see that $d x\left(\partial / \partial \xi_{1}\right)$ and $d x\left(\partial / \partial \xi_{2}\right)$ are linearly independent if and only if $\sin \beta \cos \beta \neq 0$ at $\left(\xi_{1}, \xi_{2}\right)$. Q. E. D.

LEMma 3.10. Suppose the condition (3.2) holds for any subarc $\gamma_{2}^{\prime}$ of $\gamma_{2}$ for $i=1,2$. Then a $C^{\infty}$ function $\beta\left(\xi_{1}, \xi_{2}\right)$ in Lemma 3.6 can be chosen in such a way that $0<\beta\left(\xi_{1}, \xi_{2}\right)<\frac{\pi}{2}$ for all $\left(\xi_{1}, \xi_{2}\right)$ in $R^{2}$.

Proof. By Lemma 3.7, we have

$$
\beta\left(\xi_{1}^{\prime \prime}, \xi_{2}\right)-\beta\left(\xi_{1}^{\prime}, \xi_{2}\right)=-\frac{1}{2} \int_{\xi_{1}^{\prime}}^{\xi_{1}^{\prime \prime}} \kappa_{1}\left(\xi_{1}\right) d \xi_{1} \text { for any } \xi_{1}^{\prime}, \xi_{1}^{\prime \prime}, \xi_{2}
$$

and

$$
\beta\left(\xi_{1}, \xi_{2}^{\prime \prime}\right)-\beta\left(\xi_{1}, \xi_{2}^{\prime}\right)=\frac{1}{2} \int_{\xi_{2}^{\prime}}^{\xi_{2}^{\prime \prime}} \kappa_{2}\left(\xi_{2}\right) d \xi_{2} \text { for any } \xi_{1}, \xi_{2}^{\prime}, \xi_{2}^{\prime \prime}
$$
have

Since the total curvature of $\gamma_{2}$ is zero, the condition (3.2) implies that we

$$
\left|\int_{\xi_{1}^{\prime}}^{\xi_{1}^{\prime \prime}} \kappa_{1}\left(\xi_{1}\right) d \xi_{1}\right|<\frac{\pi}{2} \text { and } \quad\left|\int_{\xi_{2}^{\prime}}^{\xi_{2}^{\prime \prime}} \kappa_{2}\left(\xi_{2}\right) d \xi_{2}\right|<\frac{\pi}{2}
$$


for any $\xi_{1}^{\prime}, \xi_{1}^{\prime \prime}, \xi_{2}^{\prime}, \xi_{2}^{\prime \prime}$.

Let $Q=\left\{\left(\xi_{1}, \xi_{2}\right): 0 \leqq \xi_{1} \leqq l_{1}, 0 \leqq \xi_{2} \leqq l_{2}\right\}$ and let $\beta_{1}=\min \left\{\beta\left(\xi_{1}, \xi_{2}\right):\left(\xi_{1}, \xi_{2}\right) \in Q\right\}$ and $\beta_{2}=\max \left\{\beta\left(\xi_{1}, \xi_{2}\right):\left(\xi_{1}, \xi_{2}\right) \in Q\right\}$. Then, by the periodicity (3.20), we see that $\beta_{1}=\min \left\{\beta\left(\xi_{1}, \xi_{2}\right):\left(\xi_{1}, \xi_{2}\right) \in R^{2}\right\}$ and $\beta_{2}=\max \left\{\beta\left(\xi_{1}, \xi_{2}\right):\left(\xi_{1}, \xi_{2}\right) \in R^{2}\right\}$. Suppose that $\beta\left(\xi_{1}^{\prime}, \xi_{2}^{\prime}\right)=\beta_{1}$ and $\beta\left(\xi_{1}^{\prime \prime}, \xi_{2}^{\prime \prime}\right)=\beta_{2}$. Then

$$
\begin{aligned}
\beta_{2}-\beta_{1} & =\beta\left(\xi_{1}^{\prime \prime}, \xi_{2}^{\prime \prime}\right)-\beta\left(\xi_{1}^{\prime}, \xi_{2}^{\prime}\right) \\
& =\beta\left(\xi_{1}^{\prime \prime}, \xi_{2}^{\prime \prime}\right)-\beta\left(\xi_{1}^{\prime}, \xi_{2}^{\prime \prime}\right)+\beta\left(\xi_{1}^{\prime}, \xi_{2}^{\prime \prime}\right)-\beta\left(\xi_{1}^{\prime}, \xi_{2}^{\prime}\right) \\
& =-\frac{1}{2} \int_{\xi_{1}^{\prime}}^{\xi_{1}^{\prime}} \kappa_{1}\left(\xi_{1}\right) d \xi_{1}+\frac{1}{2} \int_{\xi_{2}^{\prime}}^{\xi_{2}^{\prime \prime}} \kappa_{2}\left(\xi_{2}\right) d \xi_{2} \\
& \leqq \frac{1}{2}\left|\int_{\xi_{1}^{\prime}}^{\xi_{1}^{\prime \prime}} \kappa_{1}\left(\xi_{1}\right) d \xi_{1}\right|+\frac{1}{2}\left|\int_{\xi_{2}^{\prime}}^{\xi_{2}^{\prime \prime}} \kappa_{2}\left(\xi_{2}\right) d \xi_{2}\right| \\
& <\frac{\pi}{2} .
\end{aligned}
$$

We define a new function $\bar{\beta}\left(\xi_{1}, \xi_{2}\right)$ by $\bar{\beta}\left(\xi_{1}, \xi_{2}\right)=\beta\left(\xi_{1}, \xi_{2}\right)-\frac{1}{2}\left(\beta_{1}+\beta_{2}\right)+\frac{\pi}{4}$. Since $\bar{\beta}$ differs from $\beta$ by a constant, $\bar{\beta}$ also satisfies $d \bar{\beta}=\omega_{34}$. It is easy to check that $0<\bar{\beta}<\frac{\pi}{2}$. Q.E. D.

Proof of Theorem 2. By Lemma 3.9 and Lemma 3.10, a $C^{\infty}$ map $x$ becomes an immersion of a torus into $S^{3}$ in $R^{4}$ if the condition (3.2) is satisfied. (3.23) and (3.24) show that the tangent plane of the image of $x$ at each point is $e_{1} \wedge e_{2}=P\left(\xi_{1}, \xi_{2}\right)$. Hence the Gauss image of $x$ is locally the product of $\gamma_{1}$ and $\gamma_{2}$. Q.E.D.

Remark 1. Let $M$ be the image of $x$ in $R^{4}$. Since $d \omega_{12}=\omega_{13} \wedge \omega_{32}+\omega_{14} \wedge \omega_{42}$ $\equiv 0$, the Gaussian curvature of $M$ is identically zero.

Since $M$ lies in $S^{3}$, the normal connection of $M$ as a surface in $R^{4}$ is flat.

Remark 2. From the way of construction of $M$ we see that the Gauss image of $M$ is a $k$-fold covering of $\gamma_{1} \times \gamma_{2}$, where $k=1,2$ or 4 .

\section{REFERENCES}

[1] B. Y. Chen and S. Yamaguchi, Classification of surfaces with totally geodesic Gauss image, Indiana Univ. Math. J. 32 (1983), 143-154.

[2] K. Enомото, The Gauss image of flat surfaces in $R^{4}$, Kodai Math. J. 9 (1986), $19-32$.

[3] D. A. Hoffman and R. Osserman, The geometry of the generalized Gauss map, Memoirs of AMS Vol. 236 (1980).

[4] U. Pinkall, Hopf tori in $S^{3}$, Invent. Math. 81 (1985), 379-386. 
[5] H. ReckzIEgel, Completeness of curvature surfaces of an isometric immersion, J. of Diff. Geom. 14 (1979), 7-20.

[6] J. Weiner, The Gauss map for surfaces in 4-space, Math. Ann. 269 (1984), 541560 .

Faculty of Industrial Science and Technology

SCIENCE University OF TOKYo

Oshamambe, HoKkaido, 049-35, Japan 